Supplement of The Cryosphere, 12, 1195-1209, 2018

https://doi.org/10.5194/tc-12-1195-2018-supplement

(C) Author(s) 2018. This work is distributed under

the Creative Commons Attribution 4.0 License.

(c) (1)

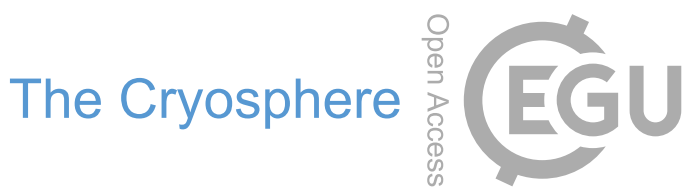

Supplement of

\title{
Climate change and the global pattern of moraine-dammed glacial lake outburst floods
}

\section{S. Harrison et al.}

Correspondence to: Stephan Harrison (stephan.harrison@exeter.ac.uk)

The copyright of individual parts of the supplement might differ from the CC BY 4.0 License. 
Despite numerous inventories of Glacial Lake Outburst Floods (GLOFs) at regional scales, no global database has been created and analyzed to place GLOFs in their wider context. This means that we are unable to answer some important questions concerning their historical behaviour and therefore the changing magnitude and frequency of GLOFs globally through time, and their likely evolution under future global climate change. This latter point is made even more difficult by the lack of longterm climate data from many mountain regions. Given the size and impacts of GLOFs in many mountain regions, better understanding their links to present and future climate change is of great interest to national and regional governments, infrastructure developers and NGOs. There is currently also a strong focus on climate change adaptation, and glacial hazard research must now be seen through this lens.

As a result, the motivation for the paper is the widely held assumption that the magnitude and frequency of GLOFs in all glaciated mountain regions is increasing in response to global climate change. The logic supporting this is that recent climate change is driving mountain glacier recession (Fig. SI 1a,b) from their late Holocene moraine limits, this is contributing to the development of proglacial and supraglacial lakes and these eventually drain catastrophically following failure of moraine dams. We wished to test this assertion. Only GLOFs associated with collapse, breaching or overtopping of moraines damming glacial lakes were counted. Such events are generally triggered by ice and rock falls or rock slides into lakes creating seiche waves or displacement waves (Reynolds and Richardson 2000). While climate change plays a dominant role in the recession of glaciers, downwasting glacier surfaces debuttress valley rock walls leading to catastrophic failure (e.g. Jiang et al 2004). Other climatically induced triggers include: increased permafrost and glacier temperatures leading to failure of ice and rock masses into lakes and the melting of ice cores in moraine dams which leads to moraine failure and lake drainage. A methodologically sound detection and attribution study needs first to formulate a hypothesis of potential impact of climate change (Clague and Evans 2000). The reasoning supporting the association between climate change and GLOFs is that climate warming results in glacier recession and glacial lake formation and evolution behind moraine dams which become unstable and fail catastrophically.

As a result, we produce a database of GLOFs developed from a collation of regional inventories (e.g. GAPHAZ and GLACIORISK databases and the GLOF Database provided under ICL ) and reviews. Only those GLOFs that could be dated to a specific year were included.

Conclusions

Given the remote nature of many of the GLOF sites, the absence of remote sensing data from the early part of the record to corroborate GLOF observations and inconsistent use of lake, glacier, region or mountain names to describe GLOFs, these and other factors complicate the creation of GLOF inventories. We are aware that some GLOFs in the record may have been given different names by different authors in inventories and this could lead to confusion. When inventories are consolidated this might, on occasion, lead to double-counting. Similar issues represent a challenge for all Detection and Attribution research on individual climatic or earth system events. As a result, all such inventories are inherently uncertain. In our inventory for consistency we have kept all GLOF records as defined by the original authors. These may be subject to future change. 
44 The takeaway points of this supplement, buttressing the points made in the main article, are that the timescales for the responses of debris-covered glaciers to climate change are long, and the limnological response timescales and GLOF trigger timescales are additional to that. Hence, climate changes that may spur lake development and outburst flooding may eventually manifest in GLOF activity a century or two centuries, or even longer, after the climatic perturbation. The GLOF record showing an upsurge in GLOF activity starting in the 1930-1950 timeframe thus probably represents a response to post-Little Ice Age warming rather than anthropogenic warming, and the decreased GLOF incidence starting late in the last century likely pertains to the stabilization of climate after the post-LIA warming but before anthropogenic warming started in earnest.

We emphasize that these results should not be construed as saying that anthropogenic climate change is somehow not affecting glacial lakes or not involved in the hazards due to them. Clearly, warming is occurring worldwide and must be affecting the growth rates of existing lakes and the inception of new ones, and some fraction-perhaps a small fraction at present-of GLOFs are triggered by recent climatic warming. We may infer that GLOF incidence rates are likely to increase later this century as anthropogenic warming takes an increasing toll on the health of glaciers worldwide.

Supplementary Information File Figures

Figure SI 1a Changes in the length of 169 glaciers worldwide from the $18^{\text {th }}$ to the $21^{\text {st }}$ century (after Oerlemans 2005). Figure SI 1b: annual change in global glacier thickness (left axis, meters of water equivalent, $\mathrm{m} / \mathrm{yr}$ ) and cumulative value (right axis, $\mathrm{m}$ ), based on surface area-weighted mass balance observations.

SI Table 1. Inventory of Glacial Lake Outburst Floods in mountain regions.

References for GLOF inventory (numbers refer to final column in SI Table)

1 GLACIORISK (2003) - http://www.nimbus.it/glaciorisk/gridabasemainmenu.aspGridbase

2 GAPHAZ

databasehttp://www.mn.uio.no/geo/english/research/groups/remotesensing/projects/gaphaz/

3 WGMS (2008) http://www.wgms.ch/

4 Raymond, M., Wegmann, M. \& Funk, M. Inventargefa"hrlicher Gletscher in der Schweiz. Mitt. VAW/ETH 182 (2003). 
5 Ives, J.D., Shrestha, R.B., \& Mool, P.K. Formation of glacial lakes in the Hindu Kush-Himalayas and GLOF risk assessment.Kathmandu: ICIMOD. (2010).

6 Reynolds,J.M . \& Richardson, S., Geological Hazards - Glacial Natural Disaster Management. A presentation to commemorate the International Decade for Natural Disaster Reduction (IDNDR) 1990-2000 (2000).

7 Reynolds, J.M. The identification and mitigation of glacier-related hazards: examples from the Cordillera Blanca, Peru. In: McCall, G.J.H., Laming, D.C.J. and Scott, S. (eds), Geo-hazards, London, Chapman \& Hall, pp. 143-157 (1992).

8 Database of glacier and permafrost disasters. University of Oslo: Department of Geosciences. (2013).

9 Reynolds, J.M. The development of a combined regional strategy for power generation and natural hazard risk assessment in a high-altitude glacial environment: an example from the Cordillera Blanca, Peru. In: Merriman, P.A. and Browitt, C.W.A. (eds), Natural Disasters: protecting vulnerable communities, London, Thomas Telford Ltd, pp. 38-50 (1993).

10 Ding, Y. \& Liu, J. Glacier lake outburst flood disasters. China Annals of Glaciology, 16, 180184 (1992).

$11 \mathrm{Xu}, \mathrm{D}$. Characteristics of debris flow caused by outburst of glacial lakes on the Boqu River in Xizang, China. Journal of Glaciololgy and Geocryology, 9( I), 23-34 (1987).

12 RGSL. Ongoing efforts to detect, monitor and mitigate the effects of GLOFs in Nepal. Project No. J9622.021. (1997).

13 Costa, J.E., \& Schuster, R.L. The formation and failure of natural dams. Geological Society of America Bulletin, 100, 1054-1068 (1988).

14 Clague J.J., Evans, S.G., \& Blown, I.G. A debris flow triggered by the breaching of a morainedammed lake, Klattasine Creek, British Columbia. Canadian Journal of Earth Sciences, 22, p. 1492-1502 (1985).

15 Clague, J.J. \& Evans, S.G. (2000) A review of catastrophic drainage of moraine-dammed lakes in British Columbia, Quaternary Science Reviews 19, 1763-1783, (2000)

16 O'Connor, J.E., Hardison, J.H., \& Costa, J.E. Debris flows from failures of Neoglacial- age moraines in the Three Sisters and Mount Jefferson wilderness areas, Oregon. US 1803 Geological Survey Professional Paper 1606 (2001).

17 RGSL. Glacial hazard assessment for the Upper Indus Basin, Pakistan, J02134 (2002). 
18 Zapata, M.L. La dinamica glaciar en lagunas de la Cordillera Blanca. Acta Montana, 19 (123), 37-60 (2002).

19 Jiang, Z.X., Cui, P., \& Jiang, L.W., Critical hydrologic conditions for overflow burst of moraine lake. Chinese Geographical Science, 14 (1), 39-47 (2004).

20 Carey, M. Living and dying with glaciers: people's historical vulnerability to avalanches and outburst floods in Peru. Global and Planetary Change 47, 122-134, (2005)

21 Kershaw JA, Clague JJ, Evans, S.G. Geomorphic and sedimentological signature of a twophase outburst flood from moraine-dammed Queen Bess lake, British Columbia, Canada. Earth Surface Processes and Landforms, 30, 1-25 (2005)

24 Narama, C., Severskiy, I., and Yegorov, A. Current state of glacier changes, glacial lakes, and outburst floods in the lle Ala-Tau and Kungoy Ala-Too ranges, northern Tien Shan Mountains, Annals of Hokkaido Geography, 84, (2010)

25 Wang, S., Zhang, M., Li, Z., Wang, F., Li, H., Li, Y. \&Huand, X. Glacier area variation and climate change in the Chinese Tianshan Mountains since 1960. Journal of Geographical Sciences, 21 263-273 (2011)

26 Worni, R., Stoffel, M., Huggel, C., Volz, C., Casteller, A. \& Luckman, B. Analysis and dynamic modeling of a moraine failure and glacier lake outburst flood at Ventisquero Negro, Patagonian Andes (Argentina). Journal of Hydrology 444/445, 134-145 (2012).

27 Fujita, K, Sakai, A, Nuimura, T, Yamaguchi, S \& Sharma, RR Recent changes in Imja glacial lake and its damming moraine in the Nepal Himalaya revealed by in situ surveys and multitemporal ASTER imagery. Environmental Research Letter 4045205 (2012).

28 Carey, M., Huggel, C., Bury, J., Portocarrero C., \& Haeberli W. An Integrated SocioEnvironmental Framework for Glacier Hazard Management and Climate Change Adaptation: Lessons from Lake 513, Cordillera Blanca, Peru, Climatic Change 112, nos. 3-4, 733-767 (2011).

29 Mergili, M. \& Schneider, J.F., Regional-scale analysis of lake outburst hazards in the southwestern Pamir, Tajikistan, based on remote sensing and GIS, Natural Hazards and Earth System Sciences, 11, 1447-1462,(2011).

30 Iribarren Anacona, P., Mackintosh, A. \& Norton, K.P. Hazardous processes and events from glacier andpermafrost areas: lessons from the Chilean andArgentinean Andes. Earth Surface Earth Surface Processes and Landforms (2014) DOI: 10.1002/esp.3524

31 URL: http://eprints.ucm.es/14013/1/MASTER_PROJECT_ClaudiaGiraldez.pdf

32 Osti, R. and Egashira, S. Hydrodynamic characteristics of the Tam Pokhari Glacial Lake outburst flood in the Mt. Everest region, Nepal. Hydrological Processes, 23, 2943-2955 (2009). 
33 RGSL. J9622.021. Ongoing efforts to detect, monitor and mitigate the effects of GLOFs in Nepal. (1997).

34 Emmer, A., Vilimek, V., Klimes, J., \&Cochachin, A. Glacier retreat, lakes development and associated natural hazards in Cordillera Blanca, Peru. In: Landslides in cold regions in context of climate change. Springer, 231-250 (2014). 
SI Figure 1

a

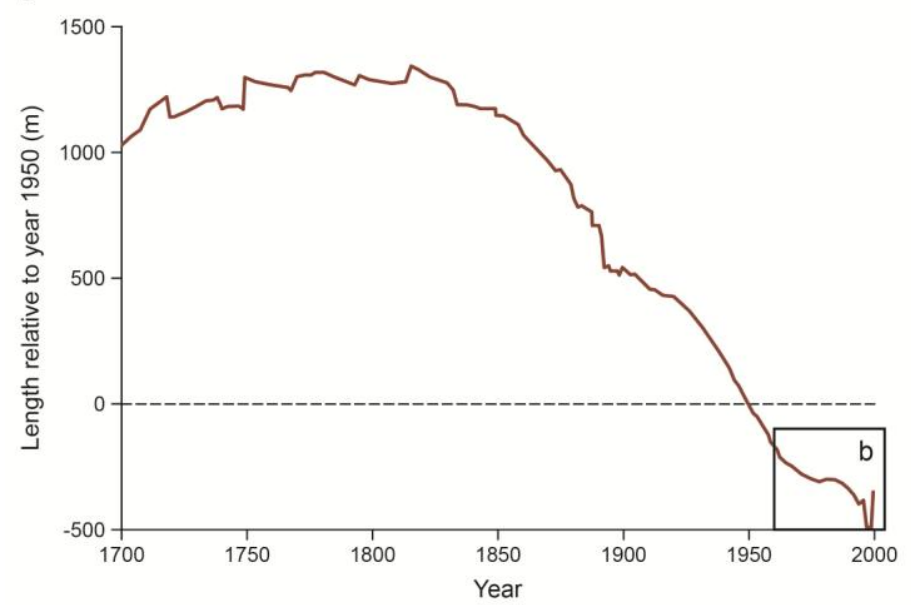

b

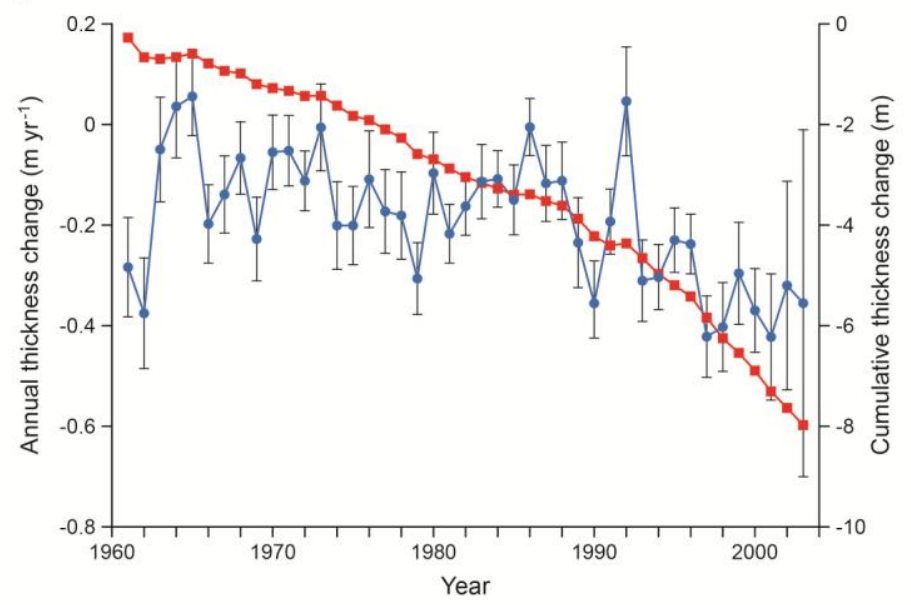

77 


\begin{tabular}{|c|c|c|c|c|c|c|c|c|c|c|c|c|}
\hline Political Unit & Site & Lake Name & Glacier or Mountain Name & Latitude & Longitude & Mth & $\mathrm{Yr}$ & \begin{tabular}{|l} 
Outburst Vol (M \\
cumecs)
\end{tabular} & $\begin{array}{l}\text { Peak Q } \\
\text { cumecs }\end{array}$ & Probable trigger & Damage & Ref. \\
\hline \multicolumn{13}{|c|}{ Europe + European Russia } \\
\hline AT & Ötztaler_Alps & & Gallruttferner & 47.05 & 10.78 & & 1890 & & & & & 1 \\
\hline AT & Ötztaler_Alps & & Winnebachferner & 47.10 & 11.05 & & 1940 & & & & & 1 \\
\hline $\mathrm{CH}$ & Western_Alps & $\mathrm{Nr}_{-} 3$ & Gruben & 46.17 & 7.97 & 8 & 1958 & & & & No damage & 4 \\
\hline $\mathrm{CH}$ & Western_Alps & $\mathrm{Nr}_{-} 3$ & Gruben & 46.17 & 7.97 & 7 & 1968 & 0.17 & & Subglacial discharge & & 3,4 \\
\hline $\mathrm{CH}$ & Western_Alps & & Sidelen & 46.50 & 8.42 & 8 & 1987 & & & Heavy rainfall & Alps and a street in the Gerental were damaged. & 4 \\
\hline $\mathrm{CH}$ & Western_Alps & Sirwolte & Griessernuhorn & 46.21 & 7.99 & 9 & 1993 & 0.30 & 90.00 & Heavy rainfall & $\begin{array}{l}\text { 10-20m high moraine breach, damage on Simplon } \\
\text { highway, destroyed gauge station. }\end{array}$ & 2 \\
\hline FR & Mont_Blanc_Massif & & Nantillons & 45.90 & 6.90 & 9 & 1944 & & & $\begin{array}{l}\text { Rapid discharge in } \\
\text { proglacial lake }\end{array}$ & $\begin{array}{l}\text { Houses in Chamonix flooded, and Montenvers railway } \\
\text { track damaged. }\end{array}$ & 1 \\
\hline IT & Piemonte & Galambra & Galambra & 45.11 & 6.85 & & 1932 & 0.50 & & & $\begin{array}{ll}\text { No damage } \\
\end{array}$ & 1 \\
\hline IT & Piemonte & & Gemelli_di_Ban & 46.40 & 8.35 & 10 & 1971 & & & & No damage & 1 \\
\hline IT & & & Mulinet S & 45.02 & 7.17 & 9 & 1993 & & & & & 2 \\
\hline IT & & & Sissone & 46.12 & 9.73 & & 1950 & & & & & \\
\hline IT & Aosta_Valley & & Trajo & 45.61 & 7.27 & & 1870 & & & & & 2 \\
\hline \multicolumn{13}{|c|}{ Hindu Kush Himayala } \\
\hline BT & Himalaya & Tarina & & 28.11 & 89.90 & & 1959 & & & & Damaged half of Punakha Dyong & 33 \\
\hline BT & Himalaya (Pho Chu) & Unnamed & & & & & 1960 & & & & & 5 \\
\hline \multirow[t]{2}{*}{$B T$} & Himalaya & Bachamancha & Bachamancha & 28.03 & 90.68 & & 1960 & & & & & 5 \\
\hline & Himalaya & & Unnamed & & & & 1968 & & & & Several houses, Punakha valley temple and Wangdi & 3 \\
\hline$B T$ & Lunana_Basin & Lugge & Lugge & 28.09 & 90.29 & 10 & 1994 & 28.00 & 2500.00 & & 26 people died \& significant property damage & 2,5 \\
\hline Bт & Lunana_Basin & & & & & & 1996 & 45.00 & & Moraine dam breach & 27 dead. Floodwave of $2 \mathrm{~m}$ at $200 \mathrm{~km}$ from source. & 6 \\
\hline BT & Lunana_Basin & Lugge & Lugge & 28.09 & 90.29 & 4 & 2009 & 1.50 & & & & 27 \\
\hline $\mathrm{CN}$ & Tibet & Zhanlonba & & & & & 1902 & & & & & 10 \\
\hline $\mathrm{CN}$ & Tibet & Tara & & 28.29 & 86.13 & 8 & 1935 & 0.60 & & \begin{tabular}{|l|l|l} 
Ice avalanche \\
\end{tabular} & $66700 \mathrm{~m}^{2}$ of wheat fields, livestocks and others damaged. & 5 \\
\hline $\mathrm{CN}$ & Tibet & Qiongbixia & & 27.83 & 88.91 & 7 & 1940 & & & Ice avalanche & Water level of Xiasim, Yadong rose 4-5m, streets flooded & 3 \\
\hline $\mathrm{CN}$ & Tibet & Sangwang & & 28.23 & 90.10 & 7 & 1954 & 5.00 & & Ice avalanche & 400 deaths in cities of Gyangze and Xigaze $200 \mathrm{~km}$ & 3,5 \\
\hline CN & Tibet & & Tara Cho (Boqu River) & 28.28 & 86.13 & 7 & 1964 & & & $\begin{array}{l}\text { Piping and moraine } \\
\text { failure }\end{array}$ & & 10 \\
\hline CN & Tibet & Damenhai & Damenhai & 29.93 & 93.15 & 9 & 1964 & 0.20 & 2812.00 & Rock avalanche & $\begin{array}{l}\begin{array}{l}\text { llocked Nyang River for }>10 \mathrm{hrs} \text {, damaged houses and } \\
\text { highway. }\end{array} \\
\end{array}$ & 3,5 \\
\hline $\mathrm{CN}$ & Tibet & Gelhaipu & Gelhaipu & 27.96 & 87.81 & 9 & 1964 & 23.36 & & Heavy rainfall & Casualties and heavy economic loss including to Chentang- & 3,5 \\
\hline $\mathrm{CN}$ & Tibet & Zhangzhangbo & & 28.06 & 86.06 & 9 & 1964 & 19.00 & 15920.00 & & No damage & 5 \\
\hline $\mathrm{CN}$ & Tibet & Longda & & 28.61 & 85.34 & 8 & 1964 & 0.50 & & Ice avalanche & & 5 \\
\hline $\mathrm{CN}$ & Tibet & Jilai & & & & 9 & 1964 & 0.50 & & Ice avalanche & & 19 \\
\hline $\mathrm{CN}$ & Tibet & Aуа & & 28.34 & 86.49 & 8 & 1965 & & & Ice avalanche & Road, bridges and others damaged. & 5 \\
\hline $\mathrm{CN}$ & Tibet & Longda & & 28.61 & 85.34 & 8 & 1968 & & & \begin{tabular}{|l|} 
Ice avalanche \\
\end{tabular} & & 3 \\
\hline $\mathrm{CN}$ & Tibet & Damenhai & Damenhai & 29.93 & 93.15 & 8 & 1968 & & & \begin{tabular}{|l|} 
Ice avalanche \\
\end{tabular} & & 3 \\
\hline $\mathrm{CN}$ & Tibet & Bugyai & & 31.77 & 94.81 & 7 & 1972 & & & Ice avalanche & & 10 \\
\hline $\mathrm{CN}$ & Tibet & Poge & & & & 7 & 1972 & & & Ice avalanche & & 5 \\
\hline $\mathrm{CN}$ & Tibet & Zhari & & & & 6 & 1981 & & & Ice avalanche & & 5 \\
\hline $\mathrm{CN}$ & Tibet & Zhangzangbo & & 28.06 & 86.06 & 7 & 1981 & 19.00 & 15920.00 & Glacier_avalanche & Damaged highway, bridge, hydropower station and & 5,11 \\
\hline $\mathrm{CN}$ & Tibet & Jzierma & & 28.08 & 86.07 & 7 & 1981 & & & Ice avalanche & & 10 \\
\hline CN & Tibet & Jin & & 28.00 & 87.16 & 8 & 1982 & 0.50 & & |Ice_avalanche & $\begin{array}{l}\text { >1600 livestock and } 280 \text { cultivated fields lost. Houses in } \\
\text { eight villages damaged. }\end{array}$ & 5 \\
\hline $\mathrm{CN}$ & Tibet & Gule & & 29.50 & 94.50 & 7 & 1988 & & & Ice avalance & & 10 \\
\hline
\end{tabular}




\begin{tabular}{|c|c|c|c|c|c|c|c|c|c|c|c|c|}
\hline $\mathrm{CN}$ & Tibet & Mitui & & & & 7 & 1988 & 0.01 & 1250.00 & Ice avalanche & & 10 \\
\hline $\mathrm{CN}$ & Tibet & Guangxie & & & & 7 & 1988 & 0.30 & & Ice avalanche & & 19 \\
\hline $\mathrm{CN}$ & Tibet & Zana & Trisuli & 28.66 & 85.37 & 6 & 1995 & & & & Destroyed $28 \mathrm{~km}$ road. & 5 \\
\hline $\mathrm{CN}$ & Tibet & & Degapu & & & 9 & 2002 & & & Ice avalanche & & 25 \\
\hline $\mathrm{CN}$ & Tibet & & Zhemalco & & & 7 & 2009 & & & Ice avalanche & & 25 \\
\hline $\mathrm{CN}$ & Tibet & & Cliaco & & & 7 & 2009 & & & Ice avalanche & & 25 \\
\hline PK & & & & & & & 1878 & & $18700 \mathrm{~m} 3$ & & & 17 \\
\hline PK & Karakoram & Karambar & Karambar & 36.62 & 74.18 & 6 & 1905 & & & 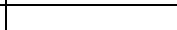 & Damage to villages above the Gilgit. Some bridges in Gilgit & 2 \\
\hline PK & Karakoram & & & & & 6 & 1967 & & & & & 17 \\
\hline PK & Karakoram & & Batura & & & & 1972 & & & & & 17 \\
\hline PK & Karakoram & & Balt Bare & & & Spr & 1974 & 5.00 & 63cumecs & & Killed 1 person. Destroyed $120 \mathrm{~m}$ bridge, part of $\mathrm{KH}$. & 17 \\
\hline $\mathrm{NP}$ & & & & & & & 1964 & & & & & 12 \\
\hline NP & Khumbu_Himal & Nagma & & 27.86 & 87.86 & 6 & 1980 & & 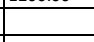 & Moraine collapse & Destroyed villages $71 \mathrm{~km}$ from source and forest. & 12 \\
\hline NP & Khumbu_Himal & & & & & 7 & 1985 & & & & & 12 \\
\hline $\mathrm{NP}$ & Khumbu_Himal & Dig & Langmoche & 27.87 & 86.58 & 8 & 1985 & 6.00 & 1600.00 & Ice avalanche & Four or five people killed. Damage to houses, HEP, & 1,2 \\
\hline $\mathrm{NP}$ & Khumbu_Himal & Chubung & Ripimosar & 27.87 & 86.45 & 7 & 1991 & 1.00 & & Ice avalanche & Na livestock killed, bridge destroyed. Beding 1 fatality, flour & 2,5 \\
\hline NP & Khumbu_Himal & NA & Amadablam & 27.81 & 86.85 & 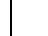 & 1993 & & & Ice avalanche & Loss of livestock and farmland and two people died. & 2,5 \\
\hline NP & Khumbu_Himal & Tam & Sabai & 27.74 & 86.84 & 9 & 1998 & 17.00 & 30000.00 & |ce avalanche & $\begin{array}{l}\text { Numerous fatalities and widespread damage. Damage } \\
\text { cost around } 2 \text { million USD. }\end{array}$ & 5 \\
\hline $\mathrm{NP}$ & Manaslu_Himal & Kabache & & 28.45 & 84.13 & 8 & 2003 & & & Moraine collapse & & 5 \\
\hline NP & Manaslu_Himal & Kabache & & 28.45 & 84.13 & 8 & 2004 & & & & & 5 \\
\hline $\mathrm{NP}$ & Himalaya & & Unnamed & 30.26 & 81.46 & 6 & 2011 & & & Ice avalanche & Damaged farmland. & 3,5 \\
\hline CA & British_Columbia & Tide & Frank_Mackie & & & 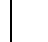 & 1929 & & & Meltwater incision & & 15 \\
\hline CA & British_Columbia & South_Macoun & & & & 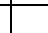 & 1949 & 0.40 & 1000.00 & & & 15 \\
\hline CA & British_Columbia & & & & & & 1965 & & & & & \\
\hline CA & British_Columbia & & Bridge & & & 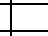 & 1967 & 2.00 & 1000.00 & & & 15 \\
\hline CA & British_Columbia & Klattasine & Homathko_Icefield & 51.17 & -124.75 & & 1972 & 1.70 & 1000.00 & Heavy rainfall & & 14 \\
\hline$C A$ & British Columbia & Cathedral & & & & L & 1978 & & & & & \\
\hline CA & British Columbia & Flood lake & & & & 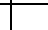 & 1979 & & & & & \\
\hline CA & British_Columbia & Peyto & & & & 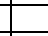 & 1983 & & & & & \\
\hline$C A$ & British_Columbia & Flood lake & & & & & 1983 & & & & & \\
\hline CA & British_Columbia & North_Macoun & & & & 7 & 1983 & & & & & 15 \\
\hline$C A$ & British_Columbia & Nostetuko & Cumberland & 51.20 & -124.40 & 7 & 1983 & 6.50 & 900.00 & |ce avalanche & Two gauging stations on Nostetuko River damaged. & 2,3 \\
\hline CA & British_Columbia & Flood lake & & & & & 1984 & & & & & \\
\hline CA & British_Columbia & Fyles & & & & 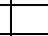 & 1984 & & & & & \\
\hline CA & British_Columbia & Tats & & & & 6 & 1990 & 0.00 & & Heavy rainfall & & 15 \\
\hline$C A$ & \begin{tabular}{|l|l|} 
British_Columbia \\
\end{tabular} & Queen_Bess & Diadem & 51.25 & -124.51 & 8 & 1997 & 8.00 & 1000.00 & Rainfall & Floodwater eroded Quaternary deposits, damaged & 2,2 \\
\hline Us & Cascade_Range & $\mathrm{NA}$ & Eugene & 44.11 & -121.78 & 8 & 1933 & & 350.00 & & & 16 \\
\hline us & Cascade_Range & Jefferson Park & NA & & & 8 & 1934 & 0.01 & 600.00 & Rapid ice melt & & 16 \\
\hline Us & Cascade_Range & & Waldo & 44.66 & -121.79 & 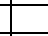 & 1937 & & & & & 16 \\
\hline Us & Sierra_Nevada_California & & Conness & 37.96 & -119.31 & 7 & 1939 & & & & & 16 \\
\hline US & Cascade_Range & & Eugene & 44.11 & -121.78 & & 1941 & & & & & 16 \\
\hline US & Cascade_Range & & & 44.12 & -121.82 & 7 & 1942 & & 360.00 & & & 13 \\
\hline US & Cascade_Range & & Collier & 44.16 & -121.77 & 7 & 1942 & 0.46 & 545.00 & & & 16 \\
\hline US & Cascade_Range & & Waldo & 44.66 & -121.79 & 7 & 1951 & & & & & 16 \\
\hline \begin{tabular}{|l|} 
us \\
\end{tabular} & Cascade_Range & & & 44.08 & -121.68 & 10 & 1966 & 0.32 & 380.00 & |ce avalanche & & 13 \\
\hline US & Cascade_Range & & & & & 9 & 1970 & 0.33 & 297.00 & & & 13 \\
\hline \begin{tabular}{|l|} 
US \\
\end{tabular} & Cascade_Range & & Diller & 44.14 & -121.77 & 9 & 1970 & 0.32 & 490.00 & & & 16 \\
\hline \begin{tabular}{|l|} 
US \\
\end{tabular} & & \begin{tabular}{|l|} 
Shoestring \\
\end{tabular} & & & & & 1980 & & & & & \\
\hline \begin{tabular}{|l|} 
US \\
\end{tabular} & \begin{tabular}{|l|} 
Cascade_Range \\
\end{tabular} & & & 44.46 & -121.83 & & 1984 & 0.01 & & & & 16 \\
\hline \begin{tabular}{|l|} 
Us \\
\end{tabular} & Alaska & \begin{tabular}{|l|} 
Peters \\
\end{tabular} & & & & & 1986 & & & & & \\
\hline \begin{tabular}{|l|} 
US \\
\end{tabular} & Alaska & Hubbard & & & & 5 & 1986 & & & & & \\
\hline \begin{tabular}{|l|} 
us \\
\end{tabular} & Alaska & Hubbard & & & & 10 & 1988 & & & & & \\
\hline \begin{tabular}{|l|} 
US \\
\end{tabular} & Alaska & $\begin{array}{l}\text { Black Rapids } \\
\end{array}$ & & & & 11 & 2002 & & & & & \\
\hline
\end{tabular}




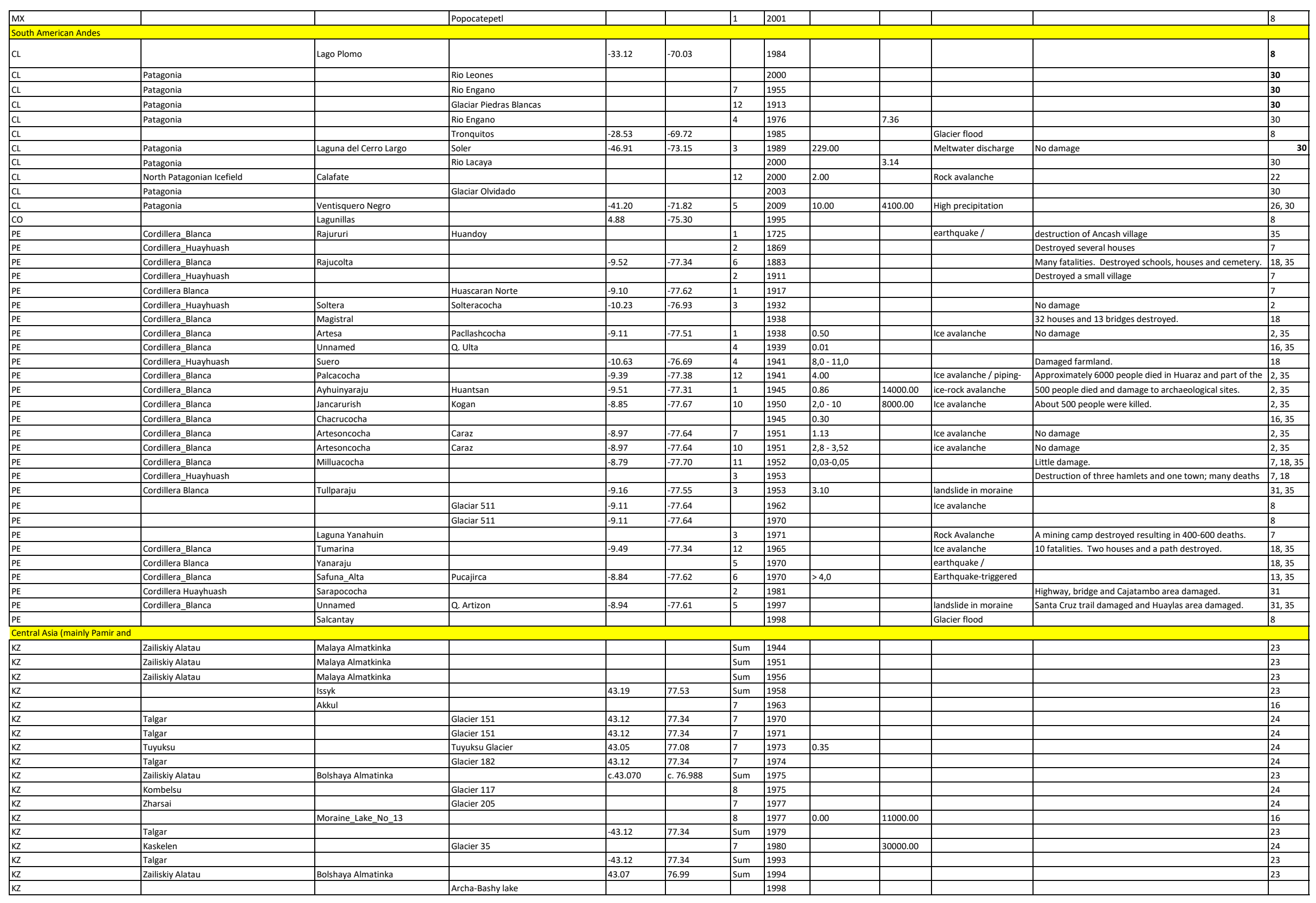




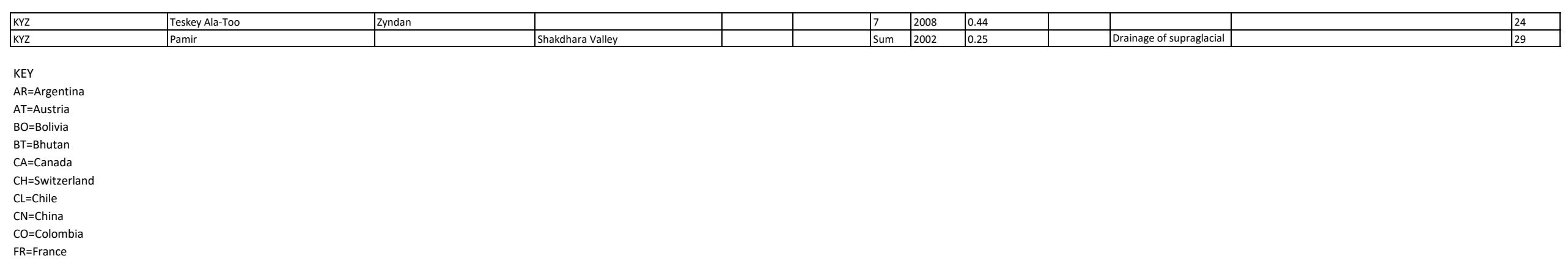

